\title{
Erratum to: Dynamic path privacy protection framework for continuous query service over road networks
}

\author{
Imran Memon ${ }^{1}$ - Qasim Ali Arain ${ }^{2}$
}

Published online: 5 December 2016

(C) Springer Science+Business Media New York 2016

\section{Erratum to: World Wide Web \\ DOI 10.1007/s11280-016-0403-3}

The original version of this article unfortunately contained a mistake. Step 3 of Algorithm 2 (Select Anonymous Region Set Generation Algorithm) was incomplete and steps 4 to 6 were missing. The corrected Algorithm 2 is provided here.

The original article was corrected.

The online version of the original article can be found at http://dx.doi.org/10.1007/s11280-016-0403-3.

Imran Memon

Imranmemon52@zju.edu.cn

Qasim Ali Arain

Qasim_ali_arain@yahoo.com

1 College of Computer Science, Zhejiang University, Hangzhou, Zhejiang, China

2 Department of Software Engineering, Mehran University of Engineering and Technology, Jamshoro, Pakistan 
Algorithm 2. Select Anonymous Region Set Generation Algorithm

1. Begin

2. The temporal-spatial queue $L_{T}$ is constructed, which each element in $L_{T}$ links a request queue.

3. The Request Enqueue Algorithm is executed to generate a new request queue $\left(\mathrm{U}_{\mathrm{id}},\langle x,, r\rangle,\left\langle R_{\min }, R_{\max }, k_{s}, t, k_{t}\right.\right.$, pre), Content,* next, *pre) and this request queue is inserted into queue $L_{T}$ in the chronological order, where $s$ and $t$ represent the space and time respectively.

4. Each element in queue $L_{T}$ is searched, and multiple threads are generated according to the condition $\left|T-T_{s}\right|<\delta$, where $T$ is the time when the element $L$ in queue $L, T$ wants to generate the request queue, $T_{s}$ is the current time of the running system, and $\delta$ is the threshold. Each request queue is assigned to a thread.

5. Multiple threads are run in parallel, and each thread is responsible for the following operations.

5.1 If the number of the elements in the request queue is smaller than $k$, the elements which satisfy the condition $\left|T-T_{S}\right|<\delta$ are searched and those elements with priority pre are deleted to form request queue set $\Omega$. When $\Omega$ is not empty, the density $\rho$ of the user is queried by the communication service provider, anonymous region $A$ and radius $R_{x m}$ with the minimum anonymous request are computed, and the anonymous request set is generated by the radius $R x m$ and the centroid of all elements in set $\Omega$. The ID disturbing algorithm is executed to disturb the ID of the user and the anonymous request set is submitted to the location services server.

5.2 If the number of the elements in the request queue is larger than $k, m$ threads are generated, where $m$ is the number of elements in the request queue. Each thread executes the following operations. 
5.3 If the three points $\left(x_{1}, y_{1}\right),\left(x_{2}, y_{2}\right)$ and $\left(x_{3}, y_{3}\right)$ in the anonymous range are located in a straight line, the coordinate of the center in the anonymous request set is $\left(x_{0}=\left(x_{1}+\right.\right.$ $\left.\left.x_{2}+x_{3}\right) / 3, y_{0}=\left(y_{1}+y_{2}+y_{3}\right) / 3\right)$; if not, the center of the circum of the triangle with coordinates $\left(x_{1}, y_{1}\right),\left(x_{2}\right.$, $\left.y_{2}\right)$ and $\left(x_{3}, y_{3}\right)$ is the center in the anonymous request set.

5.4 If $R_{x m} \leq \min \left\{R_{\max }\right\}$, a candidate anonymous region with circle center $\left(x_{0}, y_{0}\right)$ and radius $\min \{R \max \}$ is generated.

5.5 Number $s$ of the elements in the circle is computed, and the farthest point from the circle center and its distance $D$ max are recorded. If $s<k$, then report failure.

5.6 If the number of the elements which satisfy the anonymous request is also smaller than $k$, then report failure.

5.7 If $x_{j}-x_{0}>0$ and $y_{j}-y_{0}>0$ then $N_{1}=$ $N_{1}+1$, if $x_{j}-x_{0}<0$ and $y_{j}-y_{0}>0$ then $N_{2}=N_{2}+1$, if $x_{j}-x_{0}<0$ and $y_{j}$ $-y_{0}<0$ then $N_{3}=N_{3}+1$, and if $x_{j}-$ $x_{0}>0$ and $y_{j}-y_{0}<0$ then $N_{4}=N_{4}+$ $1, j=1 \sim 3$.

5.8 If each element within the circle satisfies the anonymous request, then $\Delta=\min \left\{R_{\max }\right\}-D_{\max }$ is computed. If $\Delta$ goes beyond the threshold, the radius of the circle is reduced until $\Delta$ is in the threshold. Finally, the new radius $R_{0}$ is obtained.

5.9 The anonymous region $\left(\left(x_{0}, y_{0}\right), R_{0}\right)$, the set $Q$ including all the request elements in this region and number $N_{Q}$ of the elements in set $Q$ are returned, and the density $\rho=N_{Q}$ $/\left(\pi R_{0}^{2}\right)$ of the users in the anonymous set is computed.

5.10 The ID disturbing algorithm is executed to disturb the ID of the user, set $Q$ is submitted to the location service server, and queue $L_{T}$ is renewed by the elements which are not in set $Q$ and the location indexes are updated.

\section{End}

